\title{
A Discrete Inhomogeneous Model for the Yeast Cell Cycle
}

\author{
Lucas Wardil ${ }^{\dagger \dagger}$ and Jafferson Kamphorst L. da Silva ${ }^{\dagger *}$ \\ ${ }^{\dagger}$ Departamento de Física, Universidade Federal de Minas Gerais, \\ Caixa Postal 702, CEP 30161-970, Belo Horizonte - MG, Brazil
}

(Received on 9 July, 2008)

\begin{abstract}
We study the robustness and stability of the yeast cell regulatory network by using a general inhomogeneous discrete model. We find that inhomogeneity, on average, enhances the stability of the biggest attractor of the dynamics and that the large size of the basin of attraction is robust against changes in the parameters of inhomogeneity. We find that the most frequent orbit, which represents the cell-cycle pathway, has a better biological meaning than the one exhibited by the homogeneous model.
\end{abstract}

Keywords: Protein network; Cell cycle; Dynamics

\section{INTRODUCTION}

The eukaryotic cell exhibits a common process of division into two daughter cells. This process consists of four phases [1]: (i) G1 phase, in which the cell grows; (ii) $\mathrm{S}$ phase, in which the DNA is replicated; (iii) G2 phase, that is a temporally gap between the $\mathrm{S}$ phase and the next one; (iv) $\mathrm{M}$ phase, in which the cell divides itself in two cells. In G1 phase the cell cycle rests in a stationary state until the cell size reaches a critical value and the cell receive external signals which allow it to go on the cycle. The cell-cycle regulation machinery, which controls the growth and division processes, is known for the budding yeast in detail, Saccharomyces cerevisiae $[2,3]$. In order to understand the budding yeast regulation, several models have been proposed and discussed [211]. Chen et al. [2] converted the regulation mechanism into a set of differential equations with empirical parameters. Their kinetic model has taken in account several physiological, biochemistry and genetical details. Recently, Li et al. [3] introduced a simple Boolean dynamical model to investigate the stability and robustness features of the regulatory network. They found that the cell-cycle network is stable and robust. A stochastic version of this model, controlled by a temperature like parameter, was latter studied [12]. The authors found that the stationary state and the cell-cycle pathway are stable for a wide range of the temperature parameter values. Other aspects related to the checkpoint efficiency of the Li et al. model were also considered [13].

In this work we consider the inhomogeneous version of the yeast cell-cycle introduced by $\mathrm{Li}$ et al. [3]. The cell cycle is represented by a regulatory network and the dynamics is modeled as a simple discrete dynamical system. The dynamics is constrained by the network topology by means of some parameters (coefficients) related to each network link. The link between nodes $i$ and $j$ determines the value of parameter $K_{i, j}$, which is present in the time evolution rule. If the coefficient is positive, the link represents an activation and it will be noted as $K_{i, j}=a(i, j)(a(i, j)>0)$. On the other hand, a negative coefficient $K_{i, j}=-b(i, j)(b(i, j)>0)$ represents an inactivation link. In the paper of Li et al. [3], the authors studied basically the homogeneous model $a(i, j)=b(i, j)=1$ for the nodes with a link between them. At most they have considered only two kinds of intensity $a(i, j)=a_{r}$ and $b(i, j)=a_{g}$. However, the interactions are important for the dynamics and are related to the constant rates of the kinetic equations, implying that different contributions to activation or inactivation may be present. We consider the most general inhomogeneous model of the yeast cell-cycle. Since that several different inhomogeneities represent the same dynamical model, we eliminate all possible degeneracy by constructing a minimal set of parameters (coefficients). Such set represents all kind of inhomogeneity and is very large. We find that the big basin of attraction corresponding to the stationary state is still robust to changes in the coefficients and that in the minimal set of coefficients a new orbit corresponding to the cell cycle appears more frequently having a more feasible biological significance. This means that this orbit is more robust against change in coefficients than the orbit of the homogeneous model. Moreover, the mean basin size of the global attractor is bigger than for the homogeneous case, when this more frequent orbit is present.

\section{THE DYNAMICAL MODEL}

Our model is based on the deterministic Boolean model of $\mathrm{Li}$ et al. [3] for the budding yeast cell-cycle regulation, which is represented by the regulatory network shown in Fig. 1. Each one of the 11 nodes, which represents proteins or protein complexes, is represented by a variable $S$ that takes the values 0 (the protein state is inactive) or 1 (the protein state is active). The configuration of the system at time $t$ is described by a vector $\vec{S}(t)=\left(S_{1}(t), S_{2}(t), \ldots, S_{11}(t)\right)$ that represents the state of the following proteins or complex proteins: (Cln3, MBF, SBF, Cln1-2, Cdh1, Swi5, Cdc20, Clb5-6, Sic1, Clb1-2, Mcm1). A configuration can be expressed in a short fashion by an integer $I$ if we define that $I=\sum_{i=1}^{11} S_{i} 2^{i-1}$. For example, if only Cdh1 and Sic1 are active $\left(S_{5}=1\right.$ and $\left.S_{9}=1\right)$, the related configuration $\vec{S}=(0,0,0,0,1,0,0,0,1,0,0)$ corresponds to $I(\vec{S})=272$.

The state vector $\vec{S}$ describes the cell state in a specific time. If one wants to capture the time evolution of the cell states, one needs to address a dynamical model, in which there is a time evolution rule. The configuration at time $t+1, \vec{S}(t+1)$ is related to the previous one $\vec{S}(t)$ by the relationships

$$
S_{i}(t+1)= \begin{cases}1, & \text { if } \sum_{j} K_{i, j} S_{j}(t)>0 \\ 0, & \text { if } \sum_{j} K_{i, j} S_{j}(t)<0 \\ S_{i}(t), & \text { if } \sum_{j} K_{i, j} S_{j}(t)=0\end{cases}
$$




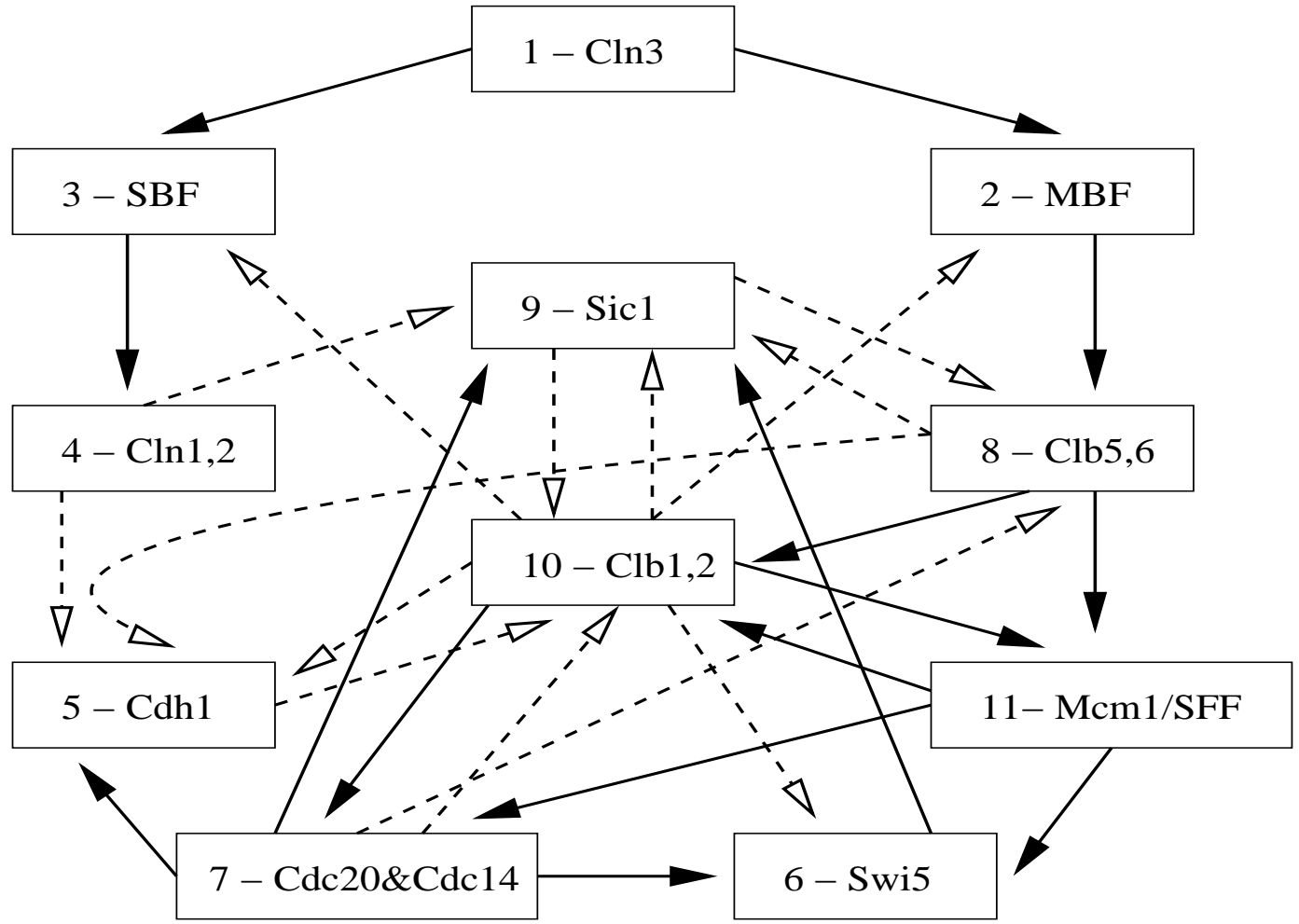

FIG. 1: The yeast cell cycle regulatory network [3]. The nodes are identified by the proteins or complex proteins and by the number used in the definition of the dynamical system. The continuous arrows mean activation and the dashed ones mean inhibition.

where $K_{i, j}$ is the interaction between nodes $i$ and $j$ and $i=$ $1,2, \ldots, 11$. If there is no link between the nodes, we have that $K_{i, j}=0$. Let us set our notation. When this link represents an activation, $K_{i, j}=+a(i, j)$. Here $a(i, j)$ is the intensity of such activation. When the interaction represents an inhibition we have that $K_{i, j}=-b(i, j)$, with $b(i, j)$ being the inhibition intensity. The nodes $1,4,6,7$ and 11 have also a time-delayed self-degradation mechanism. When $S_{i}(t)=1$ $(i=1,4,6,7,11)$ and $\sum_{j} K_{i, j} S_{j}=0$ from time $t$ to $t+t_{d}$, we will have that $S_{i}\left(t+t_{d}\right)=0$. From now on we will consider $t_{d}=1$. This evolution rule can be set in a more compact form:

$$
S_{i}(t+1)=F_{i}\left(\sum_{j} K_{i, j} S_{j}(t)\right), \text { where } F_{i}(x)= \begin{cases}1, & \text { if } x>0 \\ 0, & \text { if } x<0 \\ S_{i}(t), & \text { if } x=0\end{cases}
$$

If we name $\Omega$ as the entire state space vector, which is the space that contains the $2^{11}=2048$ possible vectors $\vec{S}(t)$, and if we name $F$ the map defined by $\vec{S}(t+1)=F(\vec{S}(t))=\left(F_{1}\left(S_{1}(t)\right), \ldots, F_{11}\left(S_{11}(t)\right)\right)$, we can define a dynamical systems as the pair $(\Omega, F)$, where $F: \Omega \rightarrow \Omega$.

From Fig. 1 we can obtain the dynamical equations. For further use, let us write these equations as the trivial ones, namely

$$
\begin{aligned}
S_{1}(t+1) & =0 \\
S_{4}(t+1) & =F_{4}\left(a(4,3) S_{3}(t)\right) \\
S_{7}(t+1) & =F_{7}\left(a(7,10) S_{10}(t)+a(7,11) S_{11}(t)\right) \\
S_{11}(t+1) & =F_{11}\left(a(11,8) S_{8}(t)+a(11,10) S_{10}(t)\right)
\end{aligned}
$$

those involving one positive and one negative links

$$
\begin{aligned}
& S_{2}(t+1)=F_{2}\left(a(2,1) S_{1}(t)-b(2,10) S_{10}(t)\right), \\
& S_{3}(t+1)=F_{3}\left(a(3,1) S_{1}(t)-b(3,10) S_{10}(t)\right),
\end{aligned}
$$


the ones involving one positive and two negative links, and the symmetrical case,

$$
\begin{aligned}
& S_{6}(t+1)=F_{6}\left(a(6,7) S_{7}(t)+a(6,11) S_{11}(t)-b(6,10) S_{10}(t)\right), \\
& S_{8}(t+1)=F_{8}\left(a(8,2) S_{2}(t)-b(8,7) S_{7}(t)-b(8,9) S_{9}(t)\right),
\end{aligned}
$$

one equation involving three negative and one positive links

$$
S_{5}(t+1)=F_{5}\left(a(5,7) S_{7}(t)-b(5,4) S_{4}(t)-b(5,8) S_{8}(t)-b(5,10) S_{10}(t)\right),
$$

and, finally, those involving three negative and two positive parameters

$$
\begin{aligned}
S_{9}(t+1) & =F_{9}\left(a(9,6) S_{6}(t)+a(9,7) S_{7}(t)-b(9,4) S_{4}(t)-b(9,8) S_{8}(t)-b(9,10) S_{10}(t)\right), \\
S_{10}(t+1) & =F_{10}\left(a(10,8) S_{8}(t)+a(10,11) S_{11}(t)-b(10,5) S_{5}(t)-b(10,7) S_{7}(t)-b(10,9) S_{9}(t)\right) .
\end{aligned}
$$

In the paper of Li et al. [3], for all links one has $a(i, j)=a_{g}$ and $b(i, j)=a_{r}$, with $a_{g}=a_{r}=1$ for the majority of results. Now we will study the general inhomogeneous case. Set new values to the coefficients means change the dynamical system definition. But will these value changes actually define new dynamical systems? Let $U$ be a subset of $\Omega$. If $F(U)=F^{\prime}(U)$ for all subset $U$, the dynamical systems $(\Omega, F)$ and $\left(\Omega, F^{\prime}\right)$ are the same. In the inhomogeneous model, the dynamical system is defined by the set $C=\left\{K_{i, j} ; 0 \leq i \leq 11,0 \leq j \leq 11\right\}$. Is that possible that a class $[C]$, which can contain more than one set of coefficients $C$, defines the same dynamical system? If this happens the non redundant inhomogeneities can be set by choosing only one element of each class $[C]$.

In order to find this minimal set we must consider two points: (i) the rule for time evolution takes into account only the sign of the sum $\sum_{j} K_{i, j} S_{j}(t)$, and (ii) the dynamical systems A and B are the same if, and only if, $\vec{S}_{A}(t+1)=\vec{S}_{B}(t+1)$ for all possible common initial condition $\vec{S}(t)$ in the state space.

We will illustrate how to find the minimal set explicitly for the dynamic of node 7 , which is in equation 1 . Note that both terms in the sum have the same sign. Whatever the values assumed by the pair $C=\{a(7,10), a(7,11)\}$, all possible initial state combinations $\left(S_{10}(t), S_{11}(t)\right)$, namely $D=\{(0,0),(1,0),(0,1),(1,1)\}$, will result on the same case (positive, negative or zero) for the sum $\sum_{j} K_{i, j} S_{j}(t)$, namely $S=\{0,+,+,+\}$. But only the sign of the sum is important for the evaluation of $S_{7}(t+1)$, and we have that all the four possible values of the pair $C$ give the same map between $S$ and $D$. If two dynamical system have the same map between $S$ and $D$ they are identical. So we have all the four possible pairs defining the same dynamical systems what means that they take part of the same class $[C]$. We only need to take a representative pair of this class, being the simplest way to choose $C=(1,1)$. For all the other nodes the procedure is the same, but we use computer programs to handle the calculations. The equation 1 have only one class, with all coefficients set equal one. The equations 2 and 3 have 3 classes, represented by $\{(1,1),(2,1),(1,2)\}$. The equation 4 and 5 have 11 classes, represented by

$\{(1,1,1),(1,1,2),(1,1,3),(1,2,1),(1,2,2),(1,3,2),(2,1,1)$,

$$
(2,1,2),(2,2,1),(2,2,3),(3,1,2)\} \text {. }
$$

The equation 6 has 67 classes and the equations 7 and 8 have 3265 classes. The minimal set that accounts for all the possible inhomogeneities has $3^{2} \cdot 11^{2} \cdot 67 \cdot 3265^{2}=7,77 \times 10^{11}$ elements. This huge quantity means that we must use a numerical simulation in order to sample this huge set.

\section{RESULTS FOR THE INHOMOGENEOUS MODEL}

First let us remind briefly the main results obtained by $\mathrm{Li}$ et al. [3] for the homogeneous model with $t_{d}=1$. From the 2, 048 initial conditions, 1,764 converge to the fixed point $I_{1}=$ 272 representing the biological $G_{1}$ stationary state. The other initial conditions converge to six fixed points $\left(I_{2}=0, I_{3}=12\right.$, $I_{4}=16, I_{5}=256, I_{6}=258$ and $\left.I_{7}=274\right)$. The pathway of the cell-cycle network is started by perturbing the $G_{1}$ stationary state: $C \ln 3$ is turned on. Then the state of the model returns to the $G_{1}$ fixed point after 12 time steps. The evolution of the proteins (or protein complexes), the so-called pathway of the cell-cycle network Path homog $=[273,278,286,14,142,1678$, $1736,1632,1888,1376,368,304,272]$, follows the biological cell-cycle sequence.

We study the inhomogeneous model by randomly choosing the coefficients from the minimal set of interactions. In a typical numerical study we have $10^{6}$ samples. For each set of coefficients we study the evolution from each one of the 2,048 initial conditions and determine the fixed points (or the rare period-2 and period-3 cycles) with its attraction basin. The $G_{1}$ fixed point 272 is always present. In our simulations we find that its minimal basin size is $B S_{\min }(272)=11$. It occurs only in $0.34 \%$ of the minimal interaction set. More rare, the maximal basin size $B S_{\max }(272)=1999$ found in our simulations, appears in $0.23 \%$ of the interactions cases. In this case, generally we have only 5 fixed points, instead of 7 found in the homogeneous model. The mean basin size of the $G_{1}$ fixed point, $B S_{\text {mean }}(272)=1622.9$, is smaller than that of the $\mathrm{Li}$ et al. model $\left(B S_{\text {homog }}(272)=1764\right)$. However, $65.3 \%$ of the interaction's minimal set have $B S(272)>1764$.

We study also the pathway of the cell-cycle network. For each sample of coefficients, the temporal evolution of the pathway begins with the perturbed state 273 , which is the sta- 
TABLE I: Orbit with initial configuration being the perturbed stationary state. The column "Time" corresponds to the time steps; the columns identified by the nodes correspond to the time evolution of this nodes; the column "Phase" corresponds to the cell cycle phases; the column "I" correspond to the integer notation for the vector states. For Cdh1 in times 8 and 9 the notation $0 / 1$ means that the left state corresponds to Path $_{\text {homog }}$ and the right one to Path $_{\text {freq }}$.

\begin{tabular}{lcccccccccccccc}
\hline Time & Cln3 & Mbf & SBF & Cln1-2 & Cdh1 & Swi5 & Cdc20 & Clb5-6 & Sic1 & Clb1-2 & Mcm1 & Phase & I \\
\hline 1 & 1 & 0 & 0 & 0 & 1 & 0 & 0 & 0 & 1 & 0 & 0 & START & 273 \\
2 & 0 & 1 & 1 & 0 & 1 & 0 & 0 & 0 & 1 & 0 & 0 & G1 & 278 \\
3 & 0 & 1 & 1 & 1 & 1 & 0 & 0 & 0 & 1 & 0 & 0 & G1 & 346 \\
4 & 0 & 1 & 1 & 1 & 0 & 0 & 0 & 0 & 0 & 0 & 0 & G1 & 14 \\
5 & 0 & 1 & 1 & 1 & 0 & 0 & 0 & 1 & 0 & 0 & 0 & S & 142 \\
6 & 0 & 1 & 1 & 1 & 0 & 0 & 0 & 1 & 0 & 1 & 1 & G2 & 1678 \\
7 & 0 & 0 & 0 & 1 & 0 & 0 & 1 & 1 & 0 & 1 & 1 & M & 1736 \\
8 & 0 & 0 & 0 & 0 & 0 & 1 & 1 & 0 & 0 & 1 & 1 & M & 1632 \\
9 & 0 & 0 & 0 & 0 & $0 / 1$ & 1 & 1 & 0 & 1 & 1 & 1 & M & 1888 \\
10 & 0 & 0 & 0 & 0 & $0 / 1$ & 1 & 1 & 0 & 1 & 0 & 1 & M & 1376 \\
11 & 0 & 0 & 0 & 0 & 1 & 1 & 1 & 0 & 1 & 0 & 0 & M & 368 \\
12 & 0 & 0 & 0 & 0 & 1 & 1 & 0 & 0 & 1 & 0 & 0 & G1 & 304 \\
13 & 0 & 0 & 0 & 0 & 1 & 0 & 0 & 0 & 1 & 0 & 0 & STATIONARY PHASE & 272 \\
\hline
\end{tabular}

tionary G1 state (272) with the Cln3 activation. Then we determine the pathway and its time step. In $10^{6}$ samples we find 336 different pathways. The pathway Path freq $=[273$, $278,286,14,142,1678,1736,1632,1904,1392,368,304$, 272] with 12 time steps is the most frequent. It appears in $10.9 \%$ of the minimal interaction set, while the pathway of the $\mathrm{Li}$ et al. model appears only in $3.6 \%$ of the cases. If we evaluate the average basin size of the $G_{1}$ fixed point in the ensemble of the cases having the most frequent pathway, we find that $B S_{\text {freq }}(272)=1866.7$. Note that this average value is large than $B S_{\text {homog }}(272)=1764$. The only difference between Path freq $_{\text {and Path }}$ homog is that in the phase M the cyclin complex Cdh1 is turned on at time step 9 in $P a t h_{\text {freq }}$ instead of time step 11 in Path homog, as it is shown in table I. Is this difference meaningful?

The exit of phase $\mathrm{M}$ is controlled by the complex APC, which is activated mainly by $\mathrm{Cdc} 20$ and $\mathrm{Cdh} 1$. The protein $\mathrm{Cdc} 20$ actives APC that begins the degradation of $\mathrm{Clb} 2$ during the transition metaphase-anaphase. The second phase of the Clb2 degradation occurs by the linking of Cdh1 with APC, during the telophase. Therefore, the $\mathrm{Clb} 2$ degradation needs a sequential action [14]: (i) Cdc20 activating APC and (ii) Cdh1 linking to APC. Although the degradation by APC-Cdc20 is enough to the exit of phase M, the degradation by APC-Cdh1 is essential to keep the G1 phase stable [15]. In the homogeneous model Cdh1 is turned on simultaneously with the turning off of $\mathrm{Clb} 2$, implying that the second degradation mechanism is absent. On the other hand, this second mechanism is present in Path $_{\text {freq }}$ because Cdh1 and Clb2 are both turned on for two time steps. We can conclude that the early activation of $\mathrm{Cdh} 1$ is more coherent with the $\mathrm{Clb} 2$ degradation mechanism.
This results show that not just the big basin of attraction of the stationary state is indeed robust against changes in the coefficients, but with inhomogeneities this basin of attraction gets bigger: $65,5 \%$ of the interaction's minimal set have basin size bigger than 1764, with a maximum value of 1999 .

\section{CONCLUSIONS}

We found that the big basin of attraction is indeed robust against change in the parameter $K_{i, j}: 65,3 \%$ of the minimal interaction set have $B S(272)>1764$ and the maximum size being 1999. Considering the orbit of the perturbed stationary state 273 we found that the most frequent orbit $\left(P_{\text {freq }}\right)$ is not the one exhibited by the homogeneous model: the orbit $P_{\text {freq }}$ appears in $10,9 \%$ of the minimum coefficient set while $P_{\text {homog }}$ appears only in 3,6\%, that is, this orbit is more robust against changes on the coefficients. Besides that, the subset of the dynamical systems exhibiting $P_{\text {freq }}$ has a mean basin size $B S_{\text {freq }}=1866,7$ that is bigger than $B S_{\text {homog }}=1764$. Not just more robust, but $P_{\text {freq }}$ has a better biological significance as we have shown by means of the earlier activation of Cdc1.

\section{Acknowledgments}

The authors thank Alcides Castro e Silva for a careful reading of the manuscript. JKLS thanks to CNPq and FAPEMIG, Brazilian agencies. LW acknowledges $\mathrm{CNPq}$ for financial support.

*To whom correspondence should be addressed (e-mail: jaff@ fisica.ufmg.br); †E-mail of LW: wardil@ fisica.ufmg.br. 
[1] B. Alberts, D. Bray, J. Lewis, M. Raff, K. Roberts, and J. D. Watson, Molecular Biology of the cell, 3rd ed., New York: Garland Publishing (1994).

[2] K. C. Chen, A. Csikasz-Nagy, B. Gyorffy, J. Val, B. Novak, and J. Tyson, Mol. Biol. 11, 369 (2000).

[3] F. Li, T. Long, Y. Lu, Q. Ouyang, and C. Tang, Proc. Natl. Acad. Sci USA 101, 4781 (2004).

[4] F. R. Cross, V. Archambault, M. Miller, and M. Klovstad, Mol. Biol. Cell 13, 52 (2002).

[5] K. C. Chen, H. C. Lee, T. Y. Lin, W. H. Li, and B. S. Chen, Bioinformatics 20, 1914 (2004).

[6] K. C. Chen, A. Calzone, Csikasz-Nagy, F. R. Cross, B. Novak, and J. J. Tyson, Mol. Biol. Cell 15, 3841 (2004).

[7] F. R. Cross, L. Schroeder, M. Kruse, and K. C. Chen, Mol.
Biol. Cell 16, 2129 (2005).

[8] B. Futcher, Curr. Opin. Cell Biol. 14, 676 (2002).

[9] A. W. Murray, Cell 116, 221 (2004).

[10] N. T. Ingolia, and A. W. Murray, Curr. Opin. Cell Biol. 14, R771 (2004).

[11] M. Tyers, Curr. Opin. Cell Biol. 16, 602 (2004)

[12] Y. Zhang, M. Qian, Q. Ouyang, M. Deng, F. Li, and C. Tang, Physica D 219, 35 (2006).

[13] G. Stoll, J. Rougemont, and F. Naef, Bioinformatics 22, 2539 (2006).

[14] F. M. Yeong, H. H. Lima, C. G. Padmashree, and U. Surana, Mol. Cell 5, 501 (2000).

[15] R. Waesch and F. R. Cross, Nature Nature 418, 556 (2002). 